\title{
An Erdős-Stone Type Conjecture for Graphic Sequences
}

\author{
John R. Schmitt ${ }^{1,2}$ \\ Department of Mathematics \\ Middlebury College \\ Middlebury, VT 05753, USA \\ Michael Ferrara ${ }^{3}$ \\ Department of Mathematical Sciences \\ University of Colorado at Denver and Health Sciences Center \\ Denver, CO 80217, USA
}

\begin{abstract}
We consider a variation of the classical Turán-type extremal problem as introduced by Erdös, Jacobson and Lehel in [4]. Let $\pi$ be an $n$-element graphic sequence and let $H$ be a graph. We wish to determine the smallest even integer $m$ such that any $n$-term graphic sequence $\pi$ having degree sum at least $m$ has some realization containing $H$ as a subgraph. Denote this value $m$ by $\sigma(H, n)$. For an arbitrarily chosen $H$, we construct a graphic sequence $\pi(H, n)$ whose degree sum plus two is at least $\sigma(H, n)$. Furthermore, we conjecture that equality holds in general, as this is the case for all choices of $H$ where $\sigma(H, n)$ is currently known.
\end{abstract}

Keywords: Degree sequence, Potentially graphic sequence 


\section{Introduction}

We consider only simple undirected graphs. We will denote the complete graph on $t$ vertices by $K_{t}$, and the join of two graphs, $G$ and $H$, by $G+H$. Further, we will write $H \subset G$ if $H$ is a subgraph of $G$ and $H<G$ if $H$ is an induced subgraph of $G$.

A sequence of nonnegative non-increasing integers $\pi=\left(d_{1}, d_{2}, \ldots, d_{n}\right)$ is said to be graphic if there exists a graph $G$ of order $n$ having degree sequence $\pi$. The graph $G$ is said to be a realization of $\pi$. Writing $\pi=\left(d_{1}^{\mu_{1}}, \ldots, d_{t}^{\mu_{t}}\right)$ will indicate that $d_{i}$ repeats $\mu_{i}$ times.

For a given graph $H$, a sequence $\pi$ is said to be forcibly H-graphic if every realization of $\pi$ contains $H$ as a subgraph. Thus Turán's classical question [22] may be re-phrased as follows: determine the least even integer $M$ so that every $n$-term graphic sequence with the sum of the terms exceeding $M$ is forcibly $K_{t}$-graphic. The same question for an arbitrary graph $H$ was considered and solved by Erdős-Stone [6] (see also Erdős and Simonovits [5]). Sometimes referred to as the fundamental theorem of extremal graph theory, their result states that this value is determined by the chromatic number of $H$ and $n$.

For a given graph $H$, a sequence $\pi$ is said to be potentially $H$-graphic if there exists some realization of $\pi$ which contains $H$ as a subgraph. In the early 1990's, Erdős, Jacobson and Lehel [4] posed the following problem: determine the least even integer $m$ so that every $n$-term graphic sequence with the sum of the terms exceeding $m$ is potentially $K_{t}$-graphic. They proposed that this value, denoted $\sigma\left(K_{t}, n\right)$, is $(t-2)(2 n-t+1)+2$ as they considered the degree sequence $\left((n-1)^{t-2},(t-2)^{n-t+2}\right)$ as the extremal sequence. (The unique realization of this sequence is $K_{t-2}+\bar{K}_{n-t+2}$.) This value was shown to be correct for the cases $t=3,4$ and 5 (see respectively [4], [11] and [16], and [17]), and $\mathrm{Li}$, Luo and Song [18] proved the conjecture true via linear algebraic techniques for $t \geq 6$ and $n \geq\left(\begin{array}{l}t \\ 2\end{array}\right)+3$. A purely graph-theoretic proof was given in [8] and also as a corollary to the main result in [3].

The aim of this note is, for an arbitrarily chosen graph $H$, to give a lower bound for $\sigma(H, n)$, that is to determine the least positive even integer so that every $n$-term graphic sequence exceeding this $\sigma(H, n)$ is potentially $H$-graphic.

\footnotetext{
1 The first author wishes to thank the Organizing Committee of the 6 th Czech-Slovak International Symposium, Prague 2006, for their generous support.

2 Email: jschmitt@middlebury.edu

3 Email: mferrara@math.cudenver.edu
} 


\section{Main Result}

We assume that $H$ has no isolated vertices and that $n$ is sufficiently large with respect to the number of vertices of $H,|V(H)|$. Let $\alpha:=\alpha(H)$ denote the independence number of $H$. We define the quantities

$$
u:=u(H)=|V(H)|-\alpha(H)-1,
$$

and

$$
d:=d(H)=\min \{\Delta(F): F<H,|V(F)|=\alpha(H)+1\} .
$$

Let $v_{i}(H)$ denote the number of vertices of degree $i$ in $H$. For all $i, d \leq$ $i \leq \alpha-1$ we define the quantity $m_{i}$ to be the minimum number of vertices of degree $i$ over all induced subgraphs $F$ of $H$ with $|V(F)|=\alpha+1$. The quantities $n_{i}, d \leq i \leq \alpha-1$ are defined recursively such that $n_{d}=m_{d}-1$ and $n_{i}=\min \left\{m_{i}-1, n_{i-1}\right\}$. Finally, we define $\delta_{\alpha-1}=n_{\alpha-1}$ and for $d \leq i \leq \alpha-2$ we define $\delta_{i}=n_{i}-n_{i+1}$.

We now consider the following sequence:

$$
\begin{array}{r}
\pi(H, n)=\left((n-1)^{u},(u+\alpha-1)^{\delta_{\alpha-1}},(u+\alpha-2)^{\delta_{\alpha-2}}, \ldots\right. \\
\left.\ldots(u+d)^{\delta_{d}},(u+d-1)^{n-u-\Sigma \delta_{i}}\right) .
\end{array}
$$

If this sequence is not graphic, then we will reduce the smallest term which is strictly greater than $u(H)$ in the sequence by one and redefine $\pi(H, n)$ to be this graphic sequence instead. Let $\sigma(\pi(H, n))$ denote the sum of the terms of $\pi(H, n)$.

Theorem 2.1 ([10]) Given a graph $H$ and $n$ sufficiently large then,

$$
\sigma(H, n) \geq \max \left\{\sigma\left(\pi\left(H^{*}, n\right)\right)+2 \mid H^{*} \subseteq H\right\} .
$$

It has been shown that equality holds in Theorem 2.1 for the following graphs: complete graphs [4], [11], [16], [17], [18], complete bipartite graphs [2],[11], [19], complete balanced multipartite graphs [8], [1], matchings [11], cycles [13], the friendship graph $\left(K_{1}+k K_{2}\right)$ [9],[12], split graphs [3], a disjoint union of cliques [7] and a complete graph with an edge deleted [14], [15]. At this time we know of no subgraph for which equality does not hold and thus conjecture that the inequality sign in Equation 1 may be replaced by an equality sign. 


\section{References}

[1] Chen, Guantao, M. Ferrara, R. Gould, and J. R. Schmitt, Graphic sequences with a realization containing a complete multipartite subgraph, submitted.

[2] Chen, G., J. Li, and J. Yin, A variation of a classical Turán-type extremal problem, European Journal of Combinatorics 25 (2004) 989-1002.

[3] Chen, G. and J. Yin, On Potentially $K_{r_{1}, r_{2}, \ldots, r_{m}}$-graphic sequences, submitted.

[4] Erdős, P., M. Jacobson, and J. Lehel, Graphs realizing the same degree sequence and their respective clique numbers, Graph Theory, Combinatorics and Applications, 1, 1991, ed. Alavi, Chartrand, Oellerman and Schwenk, 439-449.

[5] Erdős, P., and M. Simonovits, A limit theorem in graph theory, Stud. Sci. Math. Hungar. (1966), 51-57.

[6] Erdős, P., and A.H. Stone, On the structure of linear graphs, Bull. Amer. Math. Soc. 52 (1946), 1087-1091.

[7] Ferrara, M., Graphic sequences with a realization containing a union of cliques, submitted.

[8] Ferrara, M., R. Gould, and J. R. Schmitt, Potentially $K_{s}^{t}$-graphic degree sequences, submitted to Elec. J. Comb.

[9] Ferrara, M., R. Gould, and J. R. Schmitt, Graphic sequences with a realization containing a friendship graph, to appear in Ars Combinatoria.

[10] Ferrara, M. and J. R. Schmitt, A sharp lower bound for potentially H-graphic sequences, submitted to J. Graph Theory.

[11] R. Gould, M. Jacobson, and J. Lehel, Potentially G-graphic degree sequences, Combinatorics, Graph Theory, and Algorithms (eds. Alavi, Lick and Schwenk), 1, New York: Wiley \& Sons, Inc., 1999, 387-400.

[12] Lai, C., An extremal problem on potentially $K_{m}-C_{4}$-graphic sequences, submitted.

[13] Lai, C., The smallest degree sum that yields potentially $C_{k}$-graphical sequences, J. Combin. Math. Combin. Comput. 49 (2004), 57-64.

[14] Lai, C., A note on potentially $K_{4}-e$ graphical sequences, Australas. J. Combin. 24 (2001), 123-127.

[15] Li, J., R. Mao, and J. Yin, An extremal problem on the potentially $K_{r+1}-e$ graphic sequences, Ars Combinatoria 74 (2005), 151-159. 
[16] Li, J. and Z. Song, An extremal problem on the potentially $P_{k}$-graphic sequences, The International Symposium on Combinatorics and Applications, June 28-30, 1996 (W.Y.C. Chen et. al., eds.) Tanjin, Nankai University 1996, 269-276.

[17] Li, J. and Z. Song, The smallest degree sum that yields potentially $P_{k}$-graphical sequences, J. Graph Theory 29 (1998), no.2, 63-72.

[18] Li, J., R. Luo and Z. Song, The Erdös-Jacobson-Lehel conjecture on potentially $P_{k}$-graphic sequences is true, Science in China, Ser. A, 41 (1998), (5), 510-520.

[19] Li, J. and J. Yin, The smallest degree sum that yields potentially $K_{r, r}$-graphic sequences, Science in China, Ser. A, 45 (June 2002), (6), 694-705.

[20] Li, J. and J. Yin, An extremal problem on potentially $K_{r, s}$ graphic sequences, Discrete Math., 260 (2003), 295-305.

[21] Li, J. and J. Yin, Two sufficient conditions for a graphic sequence to have a realization with prescribed clique size, Discrete Math. 301 (2005) 2-3, 218-227.

[22] Turán, P., Extremalaufgabe aus der Graphentheorie, Mat. Fiz Lapook 48 (1941), 436-452. 